\title{
INTERCALATION OF FREE RADICAL, DPPH, INTO MONTMORILLONITE
}

\author{
Yuji NAKAMURA and Tokuko WATANABE \\ Laboratory of Chemistry, Tokyo University of Marine Science, \\ Konan, Minato-ku, Tokyo 108, Japan
}

\begin{abstract}
The adosorption of DPPH in the interlayer of sodium montmorillonite and sodium saponite have been investigated by ESR. As the results, DPPH molecules dimerize to form diamagnetic species in montmorillonite, whereas DPPH wolecules in saponite exist as isolated stable free radical species at room temperature.
\end{abstract}

Key words Montworillonite, Saponite, DPPH, Intercalation, ESR

\begin{abstract}
Montmorilionite and saponite are clay minerals with a layer structure, which have cations in the interlamellar space. The cations are easily exchanged with organic or inorganic cations. The inclusion phenomenon has aroused increasing interest in the field of organic and inorganic chemistry, in which it has been proved that the clay inerals have function as colum materials in liquid chromatography [1] and as supports for catalysts such as dispersed metals or metal oxides [2]. It was also suggested that a family of cyclodextrin intercalates ight be useful as artificial enzymes and as icro-encapsulating agents of unstable substances [3]. In addition, the adosorption and reaction of polar and non-polar hydrocarbons or aromatic compounds in the interlayer of clay have been extensively investigated by a wide variety of spectroscopy [4].

We choose a stable organic free radical, DPPH(1,1-diphenyl-2-picrylhydrazyl), as a guest molecule to a host compound, montmorillonite and saponite. The object of the present study is to examine an orientation and an interaction of DPPH to the surface of clay by electron spin resonance (ESR).
\end{abstract}

\section{EXPERIMENTAL}

The clays used were sodium montmorillonite with 115 meq per 1008 clay of the cation exchange capacity (CEC) and synthetic sodium saponite with 80 meq per $100 \mathrm{~g}$ clay of the CEC. They were donated by Kunimine Ind.Co., Japan. DPPH used as the adsorbate was purchased from Tokyo kasei Chem. Ind. Co., Japan. A chelate complex, bis(neocuproine)copper(1) chloride [Cu(neocup) $\left.{ }_{2}\right] \mathrm{Cl}$, used as an ion exchanger was synthesized by stoichiometrically mixing cuprous chloride with neocuproine (=neocup). Starting compounds were used without any purification. The product was recrystallized from methanol.

ESR spectra were measured to examine the adsorption properties of DPPH on clay minerals. A colloidal aqueous solution of montmorillonite of $1.00 \times$ $10^{-2} 8 / \mathrm{l}_{1}$ was added dropwise to 3 . of a methanol solution containing 8.25 $x 10^{-7}$ mol of DPPH. An aliquot of solution was transferred to a capillary sample tube for ESR measurement at each step of titration. on the other series of experiment, $0.5 \mathrm{~g}$ of each clay sample was soaked in methanol solution containing $3.3 \times 10^{-5}$ mol of DPPH and stirring with a mechanical stirrer at room temperature for several days. The adsorption of DPPH was analyzed by Languuir type plots. The suspended clay was separated by centrifugation, washed three times with methanol, centrifuged in each case and then dried in a desiccator by using rotary punp. The resultant solid was 
ground to a powder and sealed into glass capillary tubes for ESR weasurement. ESR spectra were recorded on JEOL JES-FEIX spectrometer at room temperature. Signal intensities of DPPH were normalized to a signal intensity of a standard warker, $M n^{2}+/ M g 0$. DPPH radical in methanol solution is very slowly converted into a diamagnetic species. Thus, the degradation of radical was monitored by the signal intensity of standard solution prepared at the first time of the experiment, and signal intensities of DPPH weasured in the whole process of the experiment were corrected by the signal intensity of the standard solution.

\section{RESULTS AND DISCUSSION}

Fig. 1A shows ESR spectra of DPPH in colloidal solution, and Fig.1B shows the titration curve, in which normalized ESR signal intensities of free radical, DPPH, are plotted against the amount of montmorillonite added. With increasing of the amount of montmorillonite added, signal intensity of free DPPH gradually decreases to reach to zero at the point of $7.5 \times 10^{-8}$ mol of DPPH per gram of clay. When chelate complex cations, [Cu(neocup) $]^{+}$, are introduced into the system, ESR signal intensity of free DPPH is restored as shown in Fig. 1. About $15 \%$ of the amount of adsorbed DPPH is recovered by $8.0 \times 10^{-7}$ mol of complex per gram of clay. This fact suggests that DPPH molecules are intercalated and build up diamagnetic dimers, and that the DPPH dimers intercalated in the layer are freely released into colloidal solution as monomers of DPPH, when the voluminous complex cations such as $\left[\mathrm{Cu}(\text { neocup })_{2}\right]^{+}$occupy the interlayer space by ion exchange with interlayer sodium ions. The dimerization of DPPH is similar to behavior observed for methyl viologen radical cation [5], which also have a property of dimerizing on montmorillonite surface.

(A)

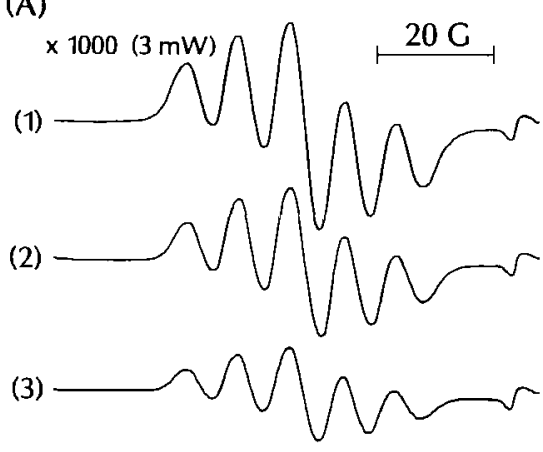

(4)

(5)

(6)

$\times 2500(5 \mathrm{~mW})$

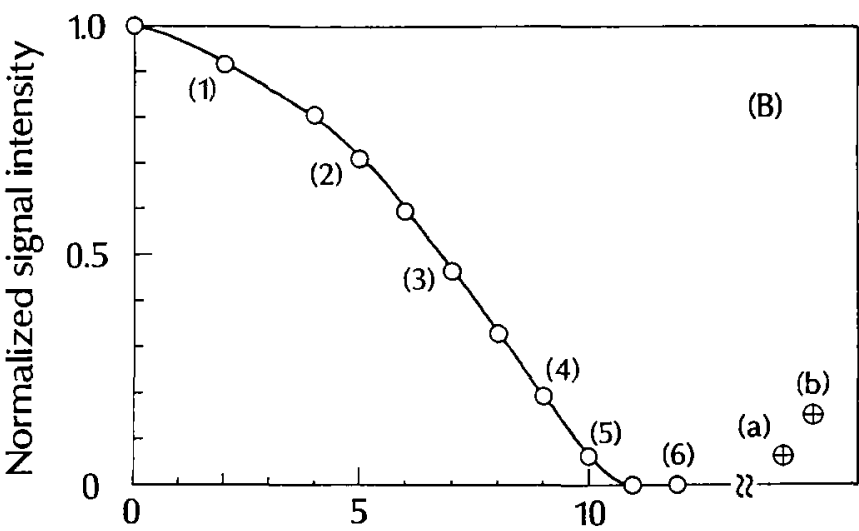

(a)

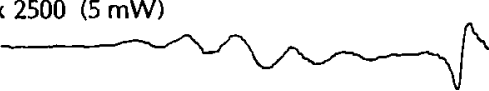

Amount of added montmorillonite / $\mathrm{mg}$

(b)

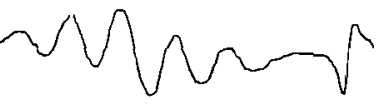

Fig. 1 DPPH-montmorillonite colloidal solution system.

(A) ESR spectra of methanol solution of DPPH including various anounts of aqueous suspension of montmorillonite (1-6) and those of colloidal solution when $4.8 \times 10^{-7} \mathrm{~mol}$ (a) and $9.6 \times 10^{-7}$ mol (b) of complex cation, $\left[\mathrm{Cu}(\text { neocup })_{2}\right]^{+}$were added to the solution of No 6 .

(B) Variation in relative intensity of ESR signals of DPPH in titrating DPPH methanol solution with a colloidal solution of montmorillonite. A total amount of $8.25 \times 10^{-7}$ mol of DPPH is normalized to unity in the absence of clay. Each number corresponds to number shown in Fig. $1 A$, respectively. $\oplus$ indicates the amount of released free radical 
A quite different phenomenon was observed in the adsorption behavior of DPPH toward saponite. DPPH molecules exist not as diamagnetic dimer, but as paramagnetic monomer in saponite layer. In order to compare the adsorption processes between montmorillonite and saponite, Langmuir type plots were depicted in Fig. 2, which shows the adsorption isotherms of DPPH on saponite and montmorillonite in a suspended condition. DPPH molecules exhibit a marked affinity towards the mineral surface. They are increasingly adsorbed with elapsed tine until the adsorbed amount reaches about $5.0 \times 10^{-5}$ mol per gram of clay in the case of montmorillonite and $2.8 \times 10^{-5}$ mol per gram of clay in the case of saponite. The adsorbed amount is about 1.8 times higher on montmorilionite than on saponite. Equilibrium was reached after about 50 hours in both system.

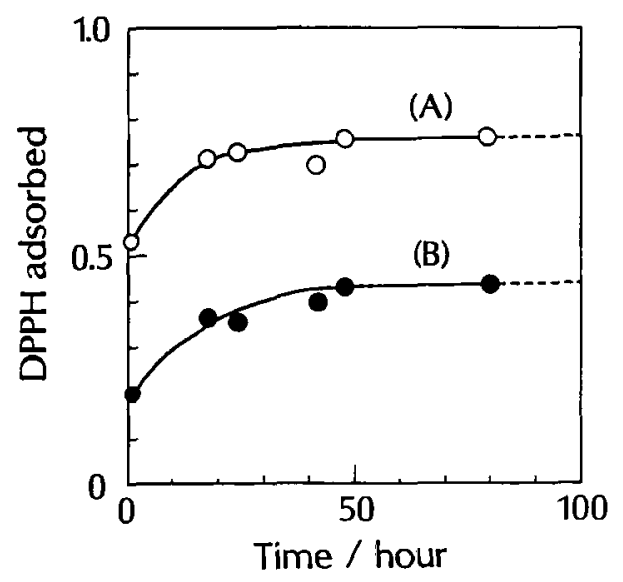

Fig. 2 Comparison of the adsorption isotherms of DPPH by (A) montmorillonite and (B) saponite dispersed in DPPH methanol solution. A value of $3.3 \times 10^{-5} \mathrm{~mol}$ of DPPH is normalized to unity.

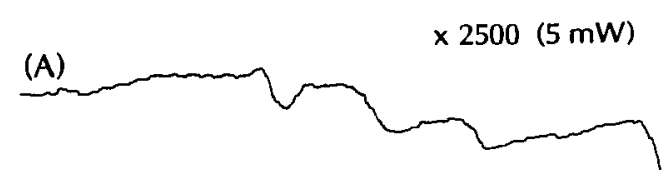

(B)

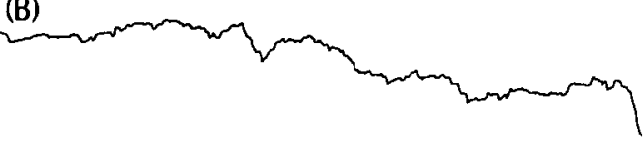

(C)
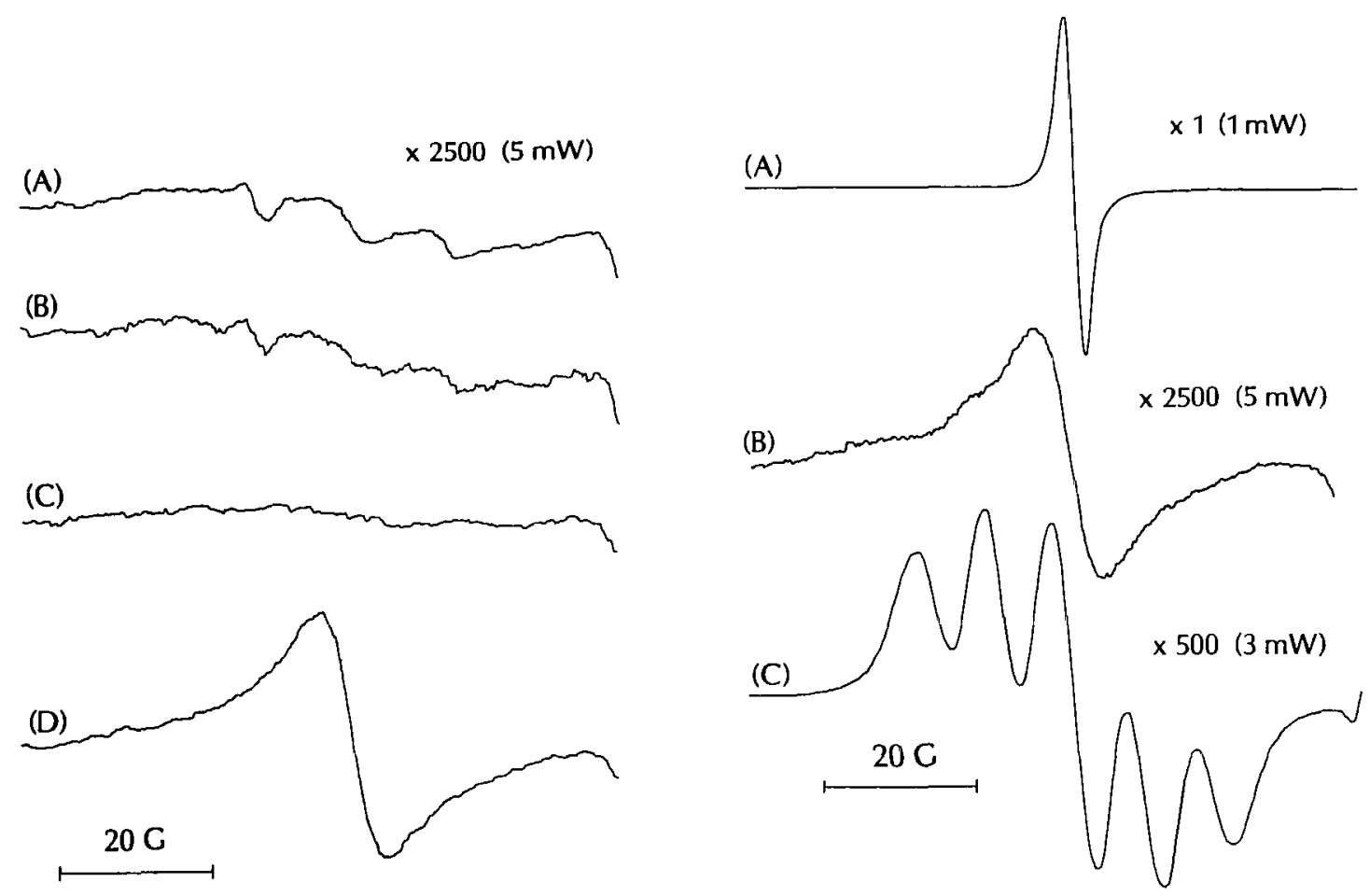

Fig. 3 ESR spectra of dried powder samples.
(A) $\mathrm{Na}$-montmorillonite
(B) Na-montworillonite intercalated DPPH
(C) $\mathrm{Na}$-saponite
(D) Na-saponite intercalated DPPH.

Fig. 4 ESR spectra of DPPH

(A) in the solid state, no clay;

(B) intercalated into $\mathrm{Na}-$ saponite;

(C) in $1.65 \times 10^{-3}$ mol/1 DPPH methanol solution. 
ESR spectra of dried powder samples are indicated in Fig. 3. No ESR signals in DPPH-montmorilionit system observed, which confirms that DPPH molecules in the interlayer of montmorilionite change diamanetic species because of dimer formation. While, in DPPH-saponite system, strong broad single line due to DPPH adsorbed on the surface was observed. As compared the broad signal by adsorbed DPPH with signals of DPPH in solid state and in wethanol solution in Fig. 4, it is suggested that DPPH molecules are distributed in the interlayer as isolatedly as they could experience no exchange narrowing. The line broadening is due to the immobilization of DPPH and possibly to the weak dipole-dipole interaction between electron spins in the interlayer

\section{CONCLUSION}

The feature of the DPPH-montworillonite system is entirely different from that of the DPPH-saponite systew. The difference seems to be casued by the distinction of the expandability which is attributed to the surface charge density and the charge localization occured by isomorphous replacement of cations in tetrahedral silicate sheets or octahedral aluminate sheets. From the results of the characterization by ESR, it is concluded that DPPH molecules for diamagnetic dimers in the interlayer space of montmorillonite, while DPPH molecules incorporated into saponite strongly interacted with the surface of silicate layer. These results suggest that the layer structure of a clay has potential as a new type of medium to arrange electron spins twodimensionally.

\section{REFERENCES}

1. Y. Nakamura, A. Vamagishi and T I wamoto, Clay Science, 8, 17 (1990). Y. Nakamura, A. Yamagishi, S. Matumoto, K. Thokubo, Y. Ohtu and M. Yamaguchi, J. Chrowatography, 462, 165 (1989).

2. P. K. Ghosh, A. W. H. Mau and A. J. Bard, J. Electroanal Chem., 169, $315(1984)$.

3. T. Kijima, H. Nakazawa and M. Kobayashi Bull. Chew. Soc. Jpn., 61, 4277 (1988).

4. B. K. G. Theng, The Chewistry of Clay-Organic Reactions, Wiley, New York, 1974 .

5. J. R. White and A. J. Bard, J. Electroanal Chem., 197, 233 (1986). 